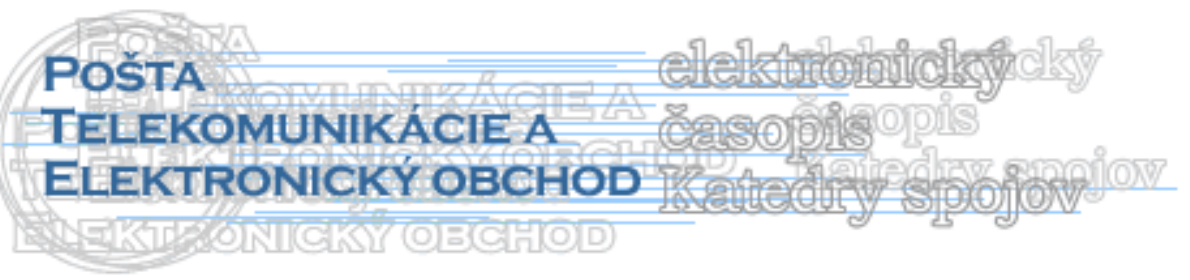

\title{
MODELLIERUNG DER ZUVERLÄSSIGKEIT VERKEHRSLOGISTISCHER NETZE
}

\author{
Reiner Keil ${ }^{1}$, Iveta Kremeňová ${ }^{2}$
}

\section{Motivation}

Für eine wissenschaftlich begründete Organisation verkehrslogistischer Prozesse ist die Herausarbeitung analytischer oder heuristischer Modelle notwendig. In dieser Arbeit soll ein Modell entworfen werden, das eine einheitliche Analyse der verkehrsorganisatorischen Prozesse ermöglicht, um daraus eine analytische Beschreibung dieser Prozesse vornehmen zu können. Als theoretisches Fundament wird dazu der von Baumann [1] auf dem theoretischen Gerüst der Logistik eingeführte Begriff der Verkehrslogistik als Organisation transportlogistischer und verkehrsinformationeller Prozesse verwendet und weiterentwickelt werden.

\section{Grundmodell zur analytischen Beschreibung verkehrslogistischer Prozesse}

Ausgangspunkt für die analytische Modellierung verkehrslogistischer Prozesse ist die Ableitung von Grundprozessen aus der allgemeinen Definition des Betreibens, wie sie in der Logistik und der Telekommunikation verwendet werden:

Betreiben $=$ Bedienen, Instandhalten und Kontrollieren

Ein verkehrslogistischer Prozess kann durch folgende, gleichzeitig und relativ unabhängig ablaufende Grundprozesse, beschrieben werden:

- $\quad$ Verkehrsobjektein- und -ausgang,

bestehend aus dem Verkehrsprozess mit den charakteristischen Zufallsgrößen

Verkehrsobjektmenge $\vec{X}=\left(\begin{array}{l}X_{E} \\ X_{A}\end{array}\right)$ und Zielfaktoren $\vec{F}=\left(\begin{array}{ccc}f_{11} & \cdots & f_{1 n} \\ \vdots & & \vdots \\ f_{n 1} & \cdots & f_{n n}\end{array}\right)$ und dem

Eingangsprozess mit der Zufallsgröße Eingangsdauer $T_{E}$ (E - Eingangszeitpunkt), der Ausgangsprozess wird nicht explizit betrachtet,

Bearbeitung, z.B. Sortieren, Verteilen oder Kommissionieren und Transport, diese Bezeichnungen sind abhängig von der Lokalisation im verkehrslogistischen Netz (Knoten oder Kante) und beinhalten das Messen und Kontrollieren, sie stehen für den Produktionsprozess, da diese Grundprozesse analytisch mit Bedienungsmodellen beschrieben werden können, werden sie hier allgemein als Bedienprozess bezeichnet

1 Dr.-Ing. Reiner Keil., Technische Universität Dresden, D - 01062 Dresden, Tel: +49-351-463-36795,

Mail: reiner.keil@tu-dresden.de

2 Doc.-Ing. Kremeňová Iveta., University of Zilina, Department of Communications, Tel: +421-41-513-

31-00, 51331 01, Mail: iveta.kremenova@fpedas.uniza.sk 
und durch die Zufallsgröße Bediendauer $T_{H}$ - bezogen auf ein Verkehrsobjekt - oder Technologiedauer $T_{Q}(\mathrm{Q}$ - Durchsatz bzw. Bedienungsrate) - bezogen auf eine Menge von X Verkehrsobjekten charakterisiert; die Technologiedauer kann auch spezifisch als Bearbeitungs-, oder Transportdauer bezeichnet werden,

- Betriebsstörung $\rightarrow$ Ausfallprozess mit der Zufallsgröße $T_{\lambda}$ Einsatzdauer $(\lambda$ Ausfallrate),

Instandhaltung $\rightarrow$ Wiederherstellungsprozess mit der Zufallsgröße Wiederherstellungsdauer $T_{\mu}$ ( $\mu$ - Reparaturrate),

darüber hinaus müssen auch die Organisationsprozesse in den Steuerungs- und Leitsystemen betrachtet werden.

Die Organisation der Betriebsprozesse in verkehrslogistischen Netzen erfolgt durch die Verbindung der Größen Prozessdauer $T_{\Pi}$ und Verkehrobjektmenge $X$. Die Prozessdauer $T_{\Pi}$ ist die von einem verkehrslogistischen Betriebssystem für die vollständige Bearbeitung bzw. dem Transport einer bestimmten Verkehrsobjektmenge $\mathrm{X}$ benötigte Zeit. Die Verkehrsobjektmenge $X$ in verkehrslogistischen Systemen ist die in einer Einheit messbare Anzahl von Verkehrsobjekten, die in einem definierten Zeitintervall bearbeitet oder vermittelt wird. Daraus wird die Verkehrsstromstärke $X_{i j}$ als die Verkehrsobjektmenge, die zwischen Punkten eines Verkehrsnetzes transportiert oder übertragen wird, abgeleitet. Die Prozessdauer $T_{\Pi}$ ist eine stochastische Größe. Sie beschreibt die für den Betriebsprozess in einem definierten Betriebssystem erforderliche Verweildauer der Verkehrsobjekte (Bearbeitungsdauer $T_{\Pi_{B}}$ im Knoten und Transportdauer $T_{\Pi_{T r}}$ auf der Kante). Bei Netzbetrachtungen entspricht die Prozessdauer $T_{\Pi}$ der Leistungszeit. Sie entspricht dem Zeitintervall, in dem eine Verkehrsobjektmenge $X$ durch ein technologisches System der Konfiguration SY in einem bestimmten Organisationsregime OR bearbeitet wird. Sie ist abhängig vom Bedienprozess, dem Ausfall- und dem Wiederherstellungsprozess. Es gilt $T_{\Pi}=f\left(T_{Q}, T_{\lambda}, T_{\mu}\right)$

Die Prozesszuverlässigkeit $Z_{\Pi}(t)$ wird definiert als die Wahrscheinlichkeit dafür, dass eine Systemkonfiguration SY bei Anwendung einer Instandhaltungsmethode IM in einer Zeitdauer $\mathrm{t}$ die zum Zeitpunkt $\mathrm{T}_{E}$ eingehende Verkehrsobjektmenge $\mathrm{X}$ vollständig bedient.

$\left.Z_{\Pi}(t)=P\left[T_{Q} \mid\left(X \mid T_{E} \wedge S Y \wedge I M\right) \leq t\right]=P\left|T_{Q}\right|\left(X\left|T_{E} \cap T_{\lambda}\right| T_{\mu} \geq t\right) \leq t\right]$

Die Prozesszuverlässigkeit wird in die folgenden Teilzuverlässigkeiten unterteilt:

- $\quad$ Bedienungszuverlässigkeit $Z_{Q}(t)$, sie beschreibt den Bedienungsprozess im Knoten und Kanten

- $\quad$ Ankunftszuverlässigkeit $Z_{A}(\tau, X)$, sie beschreibt im Zugangsbereich das Kundenverhalten und bei Verkehrsverbindungen die Auswirkungen des Transportund Übertragungsprozesses,

die Ankunftszuverlässigkeit $Z_{A}(\tau, X)$ kann in die Eingangszuverlässigkeit $Z_{E}\left(\tau_{E}\right)$ (sie beschreibt die Stochastik des Eingangszeitpunktes) und die Verkehrszuverlässigkeit $Z_{X}(x)$ (sie beschreibt die Stochastik der Verkehrsobjektmenge) aufgeteilt werden,

- $\quad$ Systemzuverlässigkeit $Z_{S y}(t)$, sie beschreibt das Ausfall- und

Wiederherstellungsverhalten, 


\section{Zuverlässigkeitstheoretische Ansätze zur Modellierung verkehrslogistischer Netze}

\subsection{Struktur des Verkehrslogistischen Netzes}

Für die analytische Modellierung verkehrslogistischer Netze werden die Ankunftsund Bedienungsprozesse getrennt und die Ausfall und Wiederherstellungsprozesse gemeinsam betrachtet. Ein verkehrslogistisches Netz kann durch das Netzmodell nach Fig. 1 beschrieben werden.

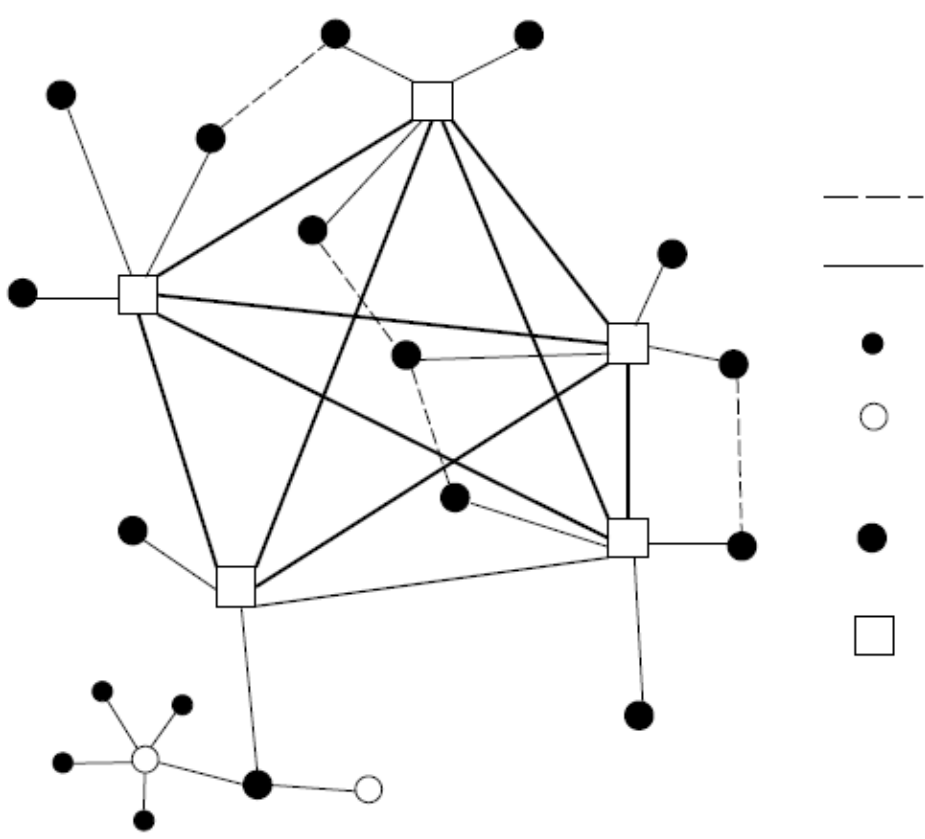

Ausnahmeverbindung

Regelverbindung

Endeinrichtung

Knoten der ersten Ebene (unterste Ebene)

Knoten der zweiten Ebene

Knoten der dritten Ebene

Fig. 1 Struktur eines verkehrslogistischen Netzes

\subsection{Ankunfts- und Bedienprozess}

Der Ankunftsprozess wird durch ein Verkehrsverteilungsmodell beschrieben. Sie dienen der Berechnung der Verkehrsströme einer Verkehrsstrommatrix eines Untersuchungsgebietes für eine bestimmte Verkehrsart. Zur Berechnung der Verkehrsströme wird benötigt:

- $\quad$ ein Verkehrsverteilungsmodell,

- $\quad$ die Quellverkehrsaufkommen $\vec{X}_{q}=\left[X_{q_{i}}\right]$,

- die Senkenverkehrsaufkommen $\vec{X}_{s}=\left[X_{s_{j}}\right]$,

- die Verkehrsstrommatrix $\vec{X}=\left[X_{i j}\right]$,

- Verkehrsnetz $G(W, E)$ mit den Gewichtsfaktoren $\vec{g}=\left[g_{i j}\right]$, diese Gewichtsfaktoren sind hier die Transportdauern $\vec{T}_{T r}=\left[T_{T_{r_{j}}}\right], \quad i, j \in W, \quad$ Transportwege $\vec{s}=\left[s_{i j}\right], \quad i, j \in W$ und weitere Bewertungsmerkmale durch die Verkehrsobjekte.

Das Verkehrsaufkommen $\vec{X}$ wird als zufälliger Vektor bzw. Matrix betrachtet. Für die Modellierung der Verkehrsverteilung wird ein entfernungsabhängiger Ansatz verwendet. Für großflächige Verkehrsnetze wird eine zeitabhängige Verkehrsquellmenge $\vec{X}_{q}$ betrachtet. Mit Hilfe eines von der Entfernung $s_{q s}$ zwischen der Quelle $w_{q}$ und der Senke $w_{s}$ abhängigen Korrekturfaktor $\eta_{E}\left(s_{q s}\right)$, der bei logistischen Prozessen einer Erlang-KVerteilung [2] unterliegt, ergibt sich 


$$
X_{q s_{\text {prog }}}(\Delta t)=X_{q}(\Delta t) \frac{X_{s}(\Delta t) \eta_{E}\left(s_{q s}\right)}{\sum_{k=1}^{\hat{k}} X_{k}(\Delta t) \eta_{E}\left(s_{k s}\right)}
$$

Die einzelnen Verkehrsströme sind zeitabhängig. In Anlehnung an die Definition der Hauptverkehrsstunde im Nachrichtenwesen wird das Berechnungsintervall auf $\Delta t=15 \mathrm{~min}$ festgelegt. Anhand dieser Verkehrsstrommatrix $\vec{X}_{\text {prog }}(\Delta t)=\left[X_{q s_{\text {prog }}}(\Delta t)\right]$ lassen sich die prognostizierten Verkehrsströme zwischen allen Verkehrsquellen und Verkehrssenken in Abhängigkeit des Zeitpunktes der Quellung darstellen. Diese Verkehrsstrommatrix wird zur aktuellen Schätzung der Verkehrsströme verwendet. In einem nächsten Schritt müssen aus der Verkehrsstrommatrix $\vec{X}_{\text {prog }}(\Delta t)$ die zeitabhängigen Verkehrsbelastungen der einzelnen Kanten berechnet werden. Aus der Gleichung (3) lassen sich die prognostizierten Zielfaktoren bestimmen. Mit den Zielfaktoren lassen sich die einzelnen Verkehrsströme bei aktuell gemessener Verkehrsquellung $\vec{X}_{q_{a k t}}$ leicht berechnen.

Ausgangspunkt der Verkehrsflussmodellierung ist die netzstrukturunabhängige und verkehrsmengenunabhängige Speicherung der Verkehrsnachfrage in der Matrix der Zielfaktoren $\vec{F}$. Die einzelnen Verkehrsobjekte müssen auf die konkrete Netzstruktur umgelegt werden. Dies erfolgt zunächst statisch ohne Berücksichtigung der Kapazitätsbeschränkungen der Kanten mit Hilfe von Wegesuchverfahren. Im nächsten Schritt, der dynamischen Modellierung, werden die Kapazitätsbeschränkungen der Knoten und Kanten insbesondere die dichteabhängige Transportgeschwindigkeit berücksichtigt. Diese Modellierungsschritte können für die 3 Ebenen [3]:

- $\quad$ Prognostizierte Verkehrsflüsse als langfristige Urdaten,

- $\quad$ mittelfristig prognostizierte Verkehrsflüsse durch Berücksichtigung von aktuellen Verkehrsmessdaten und

- $\quad$ Echtzeit-Verkehrsflussdaten zur Steuerung von Verkehrsflüssen ausgeführt werden.

\subsection{Zuverlässigkeits- und Instandhaltungsprozess}

Bei der Analyse des Ausfallprozesses wird untersucht, ob ein Verkehrs zwischen zwei Knoten in einem Netz möglich ist, d.h. mindesten ein Weg zum Transport von Verkehrsobjekten vorhanden ist. Der Ausfall von Knoten oder Kanten kann dazu führen, dass zwischen bestimmten Netzzugangsknoten zeitweilig keine Verkehrsbeziehung mehr möglich ist oder dass für die Verkehrsobjekte die Wahrscheinlichkeit dafür, einen intakten Weg zu finden, stark abnimmt. Um angeben zu können, wie zuverlässig ein Verkehrsnetz arbeitet, muss zuvor definiert werden, welche Aufgaben das Netz zu erfüllen hat und unter welchen Bedingungen das Netz als noch intakt anzusehen ist bzw. ab wann man das Netz als ausgefallen betrachten muss. Ein Verkehrsnetz ist intakt, wenn [4]:

ID1 zwischen einem ganz bestimmten Knotenpaar Verkehr möglich ist,

ID2 zwischen einer Menge bestimmter Knotenpaare Verkehr möglich ist,

ID3 Verkehr zwischen jedem Knoten aus einer Menge K mit jedem Knoten dieser Menge möglich ist,

ID4 Verkehr zwischen einem bestimmter Knoten mit jedem anderen Knoten möglich ist,

ID5 Verkehr zwischen allen Knoten möglich ist,

ID6 zwischen einem ausgewählten Knotenpaar mindestens ein vorgegebener Durchsatz bzw. eine Transport- oder Übertragungsdauer realisiert werden kann und

ID7 ein vorgegebener Durchsatz/Laufzeit zwischen jedem Knotenpaar mindestens realisiert werden kann. 
Die Netzzuverlässigkeit lässt sich durch folgende drei Modellierungsphasen charakterisieren, die sich auch in den Planungsphasen wiederspiegeln:

1. Abbildung der strukturellen Abhängigkeiten der Netzelemente untereinander bezüglich der Intaktheitsdefinition und Erfassung der relevanten Netzzustände und mögliche Übergänge zwischen ihnen (strukturelle oder deterministische Zuverlässigkeitskenngrößen). Dazu werden boolesche Modelle verwendet. Die Zuverlässigkeitsanalyse wird bei störanfälligen Knoten und/oder störanfälligen Kanten durchgeführt. Der grundsätzlich geforderte Zusammenhang einer Netzstruktur kann durch den Ausfall gewisser Knoten und Kanten verloren gehen.

2. Bewertung der Netzzustände und der möglichen Übergänge mit Hilfe der Ausfalldaten, z.B. der Verfügbarkeit der Knoten und Kanten und Ableitung der stochastischen Zuverlässigkeitsgrößen. Das Ergebnis ist die Einführung von Gewichten für Knoten und Kanten.

3. Erarbeitung von Instandsetzungsstrategien. Hierfür ist das Aufsuchen aller Minimalwege unter der Berücksichtigung der strukturellen Importanzen zur Reihenfolgeentscheidungsfindung erforderlich.

Deterministische Zuverlässigkeitskenngrößen geben den erforderliche Aufwand an, um ein Netz vom intakten Zustand in eine ausgefallenen Zustand zu überführen. Bei der Bestimmung deterministischer Kenngrößen werden strukturelle Schachstellen im Netz in Abhängigkeit von der Intaktheitsdefinition und den technologischen Besonderheiten aufgedeckt. Da diese Kenngrößen das Ausfallverhalten der Netzelemente nicht berücksichtigen, geben sie keine Informationen darüber, wie häufig und wie lange das Netz seine Aufgaben nicht erfüllt.

Stochastische Kenngrößen geben z.B. an:

- $\quad$ die Wahrscheinlichkeit dafür, dass das Netz zu einem bestimmten Zeitpunkt intakt ist,

- $\quad$ die mittlere Anzahl der Netzausfälle in einem bestimmten Zeitpunkt oder

- $\quad$ den mittleren Anteil eines Zeitintervalls, während dem das Netz intakt ist.

Die stochastischen Kenngrößen werden ebenfalls in Abhängigkeit von einer Intaktheitsdefinition bestimmt, wobei neben der Netztopologie vor allem die Angaben über das Ausfallverhalten der Netzelemente eingehen.

Die Bestimmung der einfachsten Sicherheitskenngrößen eines Netzes kann bereits auf der Basis unbewerteter Graphen durchgeführt werden. Dabei wird der Ausfall eines Netzelementes mit dem Entfernen der entsprechenden Kante oder des Knoten aus dem Graphen gleichgesetzt. Bei einem Knotenausfall müssen zusätzlich alle mit diesem Knoten adjazenten Kanten entfernt werden. Die deterministischen Kenngrößen geben an, wie viele Netzelemente entfernt werden müssen, damit die Intaktheitsbedingung nicht mehr erfüllt wird. Die minimale Anzahl von Kanten, die aus dem Graphen G entfernt werden müssen, um alle Wege vom Quellknoten $w_{q}$ zum Senkeknoten $w_{s}$ zu unterbrechen, wird mit $\zeta_{E}\left(w_{q}, w_{s}\right)$ bezeichnet und heißt Kantenzusammenhang zwischen $w_{q}$ und $w_{s}$. Der Kantenzusammenhang ist eine einfache deterministische Zuverlässigkeitskenngröße für Netze mit der Intaktheitsdefinition ID1. Eine der wichtigsten Kenngrößen für ein Verkehrsnetz ist die Netzverfügbarkeit. Sie ist die Wahrscheinlichkeit dafür, dass sich das Netz zu einem ausgewählten Zeitpunkt im funktionsfähigen Zustand befindet.

Zuverlässigkeitsersatzschaltungen für Verkehrsnetze werden unter Anwendung des booleschen Zustandsmodells abgeleitet. Dazu wird die Systemfunktion $\varphi$ mittels Zuverlässigkeitsersatzschaltung bezüglich der Intaktheit beschrieben. Der Zustand des Systems ist eine boolesche Funktion der booleschen Variablen $\left(x_{1}, \ldots, x_{n}\right)$. Es gilt

$\varphi\left(x_{1}, \ldots, x_{n}\right)=1$

falls das System funktioniert und 
$\varphi\left(x_{1}, \ldots, x_{n}\right)=0$

falls das System ausgefallen ist. Für die weitere Betrachtung genügt es, nur bestimmte Zustandsvektoren zu verwenden, diese können als Minimalweg $\psi_{\min }\left(w_{q}, w_{s}\right)$ determiniert werden. Fällt im Minimalweg mindestens eine Kante aus, existiert zwischen Quelle und Senke keine Verbindung mehr. Zusammenhänge zwischen Elementeausfällen und Systemausfällen werden durch Zuverlässigkeitsersatzschaltungen (ZES) verdeutlicht.

Praktisch relevant sind folgende Fälle [4]:

1. Zwischen den Knoten $w_{q}$ und $w_{s}$ existiert nur ein Weg, dies ist auch der Minimalweg $\psi_{\text {min }}\left(w_{q}, w_{s}\right)$, z.B. bei planmäßigen Transportprozessen,

2. Zwischen den Knoten $w_{q}$ und $w_{s}$ existiert nur wenige Weg, z.B. bei verbindungsorientierten Übertragungsnetzen in der Telekommunikation, d.h. es existieren Kanten $e \in E$, die zu keinem Minimalweg gehören,

3. Zwischen den Knoten $w_{q}$ und $w_{s}$ existieren sehr viele Weg, z.B. bei verbindungslosen Übertragungsnetzen in der Telekommunikation, d.h. alle Kanten $e \in E$ gehören zu mindestens einem Minimalweg.

Eine bewertungsminimale Instandsetzung gestörter Verkehrsnetze macht es erforderlich, die Netzkanten zusätzlich zu bewerten. Im allgemeinen werden bei der Anwendung von Netzmodellen die Netzelemente mit speziellen Charakteristika versehen (z.B. Länge, Kosten, Zuverlässigkeit, Instandsetzungsaufwand, Arbeitskräfteaufwand, Zeitdauer der Instandsetzung), den so genannten Gewichten (Knotengewichten, Kantengewichten) $g_{k}$, die eine fiktive Gewichtsgröße der Kante $e_{k} \in E, g_{k} \in \aleph$ darstellt.

\section{Modellansatz für eine Instandsetzungsstrategie}

Verkehrsnetze können verschiedene deterministische und stochastische Zustände annehmen, die sich gegenseitig bedingen. Es können hier folgende Netzbetriebszustände definiert werden [3]:

- $\quad$ Nennbetrieb: Der Netzgraph $G(W, E)^{n e n n}$ befindet sich im Nennbetrieb, seine Netzelemente sind intakt.

- Störbetrieb: Der Netzgraph $G(W, E)^{\text {stör }}$ befindet sich im Störbetrieb oder auch im minimal instandgesetzten Zustand. Die einfache Netzverbundenheit ist dahingehend wieder hergestellt, dass jede Quelle-Senke-Beziehung über einen Weg $\psi\left(w_{q}, w_{s}\right)$ verbunden ist. Dieser Netzzustand wird auch als Zwischenzustand bezeichnet. Die Zielstellung der langfristigen Instandsetzung besteht in der endgültigen Wiederherstellung des Verkehrsnetzes. Aus dem Netzgraph $G(W, E)^{\text {stör }}$ wird durch Kanteninstandsetzung der Netzgraph $G(W, E)^{\text {nenn }}$.

- Kollaps: Der Netzgraph $G(W, E)^{\text {koll }}$ befindet sich im Kollaps. Er stellt das Modell des gestörten Verkehrsnetzes dar. Die Kantenmenge $e_{k}^{\text {koll }}$ ist zerstört und muss instand gesetzt werden.

Hinsichtlich der Instandsetzungsstrategien lassen sich 3 Arten charakterisieren [4]:

1. Instandsetzungsstrategie des präventiven Schutzes: hierbei geht es um den Erhalt des Nennbetriebes,

- $\quad$ rechnerisch geschieht dies durch Bilden der dualen Menge sämtlicher minimaler Schnitte aus den Minimalwegen einer Struktur, 
2 Minimalinstandsetzung:

- $\quad$ hierbei geht es um den Übergang vom Kollaps zur unteren Grenze des Nennbetriebes, wo je nach Intaktheitsdefinition effektiv der Netzzustand hergestellt wird, damit diese Intaktheitsdefinition erfüllt wird,

- $\quad$ rechnerisch geschieht dies über die Bestimmung der Ausfallhäufigkeiten der Netzkanten bei Kenntnis aller Minimalwege,

3. Langfristige Instandsetzung:

- $\quad$ hierbei geht es um den Übergang vom Störbetrieb zum Nennbetrieb, die Kenntnisse sind aber auch bei der Netzplanung zur Schaffung eines Optimalbetriebes relevant,

- $\quad$ rechnerische Methoden sind das Bestimmen zuverlässiger kantendisjunkter Übertragungswege aus den Minimalwegen und

- $\quad$ stochastische Berechnungen der Verbindungsverfügbarkeiten auf Grundlage von ZES, bei denen Elemente mehrfach vorkommen.

Die Grundgedanken für entsprechende Ansätze sind:

- Welche Kanten zwischen den Knotenmengen des Netzes sind aufgrund der konkreten Netzstruktur besonders anfällig für einen Ausfall?

Welche Kanten des Netzes sind aufgrund einer konkreten Netzstruktur besonders anfällig für einen Ausfall?

Welche Ersatzwege müssen instandgesetzt oder vorgesehen werden?

\subsection{Bestimmung der Prozesszuverlässigkeit}

Für die Berechnung der Prozesszuverlässigkeit eines Verkehrsnetzes existieren unterschiedliche Wege:

1. Bestimmung der Teilzuverlässigkeiten (technologische Zuverlässigkeit und Systemzuverlässigkeit) für das gesamte Netz und daraus die Netzprozesszuverlässigkeit oder

2. die Bestimmung der Netzprozesszuverlässigkeit aus den Prozesszuverlässigkeiten der einzelnen Knoten und Kanten.

Bei der Anwendbarkeit analytischer Algorithmen muss unterschieden werden, ob das verkehrslogistische Netz ohne Verbindungsredundanz (hier erfolgt der Betrieb z.B. in der Logistik nach einem Fahrplan) oder mit Verbindungsredundanz (hier erfolgt ein dispositiver Betrieb) organisiert wird. Außerdem muss der Wirkungsbereich der Prozesszuverlässigkeit beachtet werde. Es können z.B. einzelne oder einige Relationen, ein bestimmtes Gebiet oder das ganze Netz betrachtet werden.

Für verkehrslogistische Netze ohne Verbindungsredundanz kann die Prozesszuverlässigkeit einer Relation $\rho\left(w_{q}, w_{s}\right)$ mit hinreichender Genauigkeit analytisch berechnet werden. Unter Beachtung der Unabhängigkeit der einzelnen Netzelemente kann die Prozesszuverlässigkeit des Netzes aus dem Produkt der Prozesszuverlässigkeit der einzelnen Knoten und Kanten des Weges $\psi\left(w_{q}, w_{s}\right)$, die für eine Relation $\rho\left(w_{q}, w_{s}\right)$ nach dem Fahrplan verwendet werden, berechnet werden. Es gilt

$$
Z_{\Pi}(t)=\prod_{e, w \in \psi\left(w_{q}, w_{s}\right)} Z_{\Pi_{e, w}}(t)
$$

Bei der Berechnung der Prozesszuverlässigkeit des verkehrslogistischen Netzes kann nicht mehr von der Unabhängigkeit der einzelnen Netzelemente ausgegangen werden, dies gilt insbesondere bei der Berechnung der technologischen Zuverlässigkeit. Hierfür müssen andere Ansätze gewählt werden, die bei der Betrachtung verkehrslogistischer Netze mit Verbindungsredundanz verwendet werden. Zur Berechnung der Prozesszuverlässigkeit von 
verkehrslogistischen Netzen mit Verbindungsredundanz existieren derzeit keine analytischen Lösungen. Kernpunkt eines heuristischen Ansatzes ist die Bestimmung der Infrastrukturzuverlässigkeit des verkehrslogistischen Netzes anhand der Intaktheitsdefinition ID7. Dazu muss die zugehörige Strukturfunktion $\phi_{Q_{\min _{w_{q}, w_{s}}}}$ bestimmt werden. Der minimale Pfadvektor sind dann die minimalen Weg von $w_{q_{i}}$ nach $w_{s_{i}}$, die jeweils den Durchsatz $Q_{\text {min }_{i}}$ realisieren können. Ein Weg für die Einbeziehung der technologischen Prozesse ist die Transformation der Kantenverfügbarkeit $V_{S y_{e}}$ in Abhängigkeit der technologischen Zuverlässigkeit. Dieser Weg kann auch bei der Betrachtung der anderen Intaktheitsdefinitionen verwendet werden. Damit kann über den Weg der Berechnung der Systemzuverlässigkeit die Prozesszuverlässigkeit abgeschätzt werden. Dem Autor sind zur Lösung dieses Problems jedoch keine analytischen Ansätze bekannt. Ein Ausweg kann der Einsatz der Simulation sein.

\section{4. $\quad$ Fazit und Ausblick}

Über die dargestellten Modelle und Algorithmen lassen sich einfache logistische Systeme analytisch beschreiben. Für die Analyse vernetzter Strukturen konnten daraus Ansätze abgeleitet werden, um Knoten, Kanten oder einzelne Verkehrsrelationen mathematisch zu beschreiben und es wurden Grundlagen geschaffen, um komplexe Netze numerisch zu modellieren. Mit diesen Modellen wurde ein Grundgerüst zur Gestaltung, Dimensionierung und Optimierung solch komplexer Systeme geschaffen, welches durch gezielten Einsatz weiterer Methoden angepasst und optimiert werden kann. Dabei erschließt sich natürlich auch für Teilprozesse, die sich bisher einer analytischen Beschreibung entziehen, der Einsatz der Simulation. Ebenso liefert insbesondere die moderne „online“ Simulation Möglichkeiten, die bei einer Analytik auftretenden Probleme der Randbedingungen bzw. Einschränkungen, die dazu noch sehr dynamisch sein können, fundiert zu überwinden. Gelingt es in der Zukunft, analytische Verfahren und Simulation zur Dimensionierung logistischer Systeme und Prozesse sinnvoll zu integrieren, so ist das Ziel einer automatisierten Produktion für den stofflichen Bereich der Verkehrslogistik möglich.

\section{Literatur}

[1] BAUMANN, S.: Organisation von stofflichen und informellen Verkehrsprozessen, Habilitation, Universität Zilina (Sk), 2006

[2] KEIL, R.: Analytische Ansätze zur Modellierung verkehrslogistischer Prozesse - Teil 1 Analyse, in Schriftenreihe des Sächsischen Telekommunikationszentrum e.V. Band 11/2011, Dresden 2011, ISSN 1863-1787

[3] KEIL, R.: Analytische Ansätze zur Modellierung verkehrslogistischer Prozesse - Teil 2 Modellierung, in Schriftenreihe des Sächsischen Telekommunikationszentrum e.V. Band 12/2011, Dresden 2011, ISSN 1863-1787

[4] FUß, T.: Betrachtungen zur Netzzuverlässigkeit des CNI-Netzes in Deutschland, Firmenschrift CNI 1995

[5] BADER,T.,KREMEŇOVÁ,I.: Kundenzufriedenheit durch Qualitätsmanagementsysteme, Posta, Telekomunikation und E-commerce, Zilinaer University, ISSN 1336-8281, 3/2008

"Article was created by support of the following grant projects: VEGA 1/0199/11- Research about interoperability of strategic management methods with the intention to Organizations, and KEGA 052ŽU-4/2012 On-line learning management in education in the field of information and communication technologies.“ 